\title{
MELHORIA NA EFICIÊNCIA DOS EXPERIMENTOS DE INDICAÇÃO DE CULTIVARES DE MILHO
}

\author{
Enhanced efficicency in experiments for maize cultivar indication
}

\author{
Edilson Romais Schmildt ${ }^{1}$, Willian Krause ${ }^{2}$, Cosme Damião Cruz ${ }^{3}$
}

\begin{abstract}
RESUMO
Objetivou-se com este trabalho verificar o comportamento da precisão experimental mediante o uso de métodos de correção de produtividade das parcelas experimentais e suas influências sobre a comparação e indicação de cultivares de milho pelo método de Annicchiarico (1992). Foram analisadas 33 cultivares com três repetições em blocos casualizados em 8 ambientes, submetidos à correção do rendimento de parcelas pelos métodos da covariância para estande ideal (COVI) e pela correção estratificada (CE), incluindo a análise sem correção (SC). Houve redução no coeficiente de variação, com o ajuste da produtividade, melhorando a eficiência dos experimentos. Os métodos COVI e CE foram consistentes na correção da produtividade para indicação de cultivares. A correção dos dados influencia na indicação de cultivares.
\end{abstract}

Termos para indexação: Zea mays L., precisão experimental, análise estatística, indicação de cultivar, correção de produção.

\begin{abstract}
The objective of this study was to assess the experimental precision when using correction methods for experimental plot yields and the influences of these methods on the comparison and indication of maize cultivars by the method of Annicchiarico (1992). Thirty-three cultivars were evaluated in three replications in randomized blocks in eight environments, with plot yields being analysed without correction (SC) and corrected by the methods of covariance to the ideal stand (COVI) and by the stratified correction (CE). There was a reduction in the coefficient of variation with the yield adjustment, improving the efficiency of the experiments. The methods COVI and CE were consistent in the correction of the yield for the indication of cultivars. The data adjustment affected the cultivar indication.
\end{abstract}

Index terms: Zea mays L.; experimental precision; statistical analysis; cultivar indication; yield correction.

(Recebido para publicação em 22 de setembro de 2004 e aprovado em 1 de setembro de 2005)

\section{INTRODUÇÃO}

Devido a dinâmica dos programas de melhoramento, tanto das empresas públicas como privadas, a cada ano são colocadas no mercado dezenas de cultivares de milho. No Brasil, o milho é cultivado em uma diversidade grande de ambientes, que podem ocorrer em função da variação de locais, das épocas de semeadura, dos níveis de adubação, das questões sociais, técnicas e econômicas, entre outras.

As cultivares de milho comercializadas nas diversas regiões do País nem sempre são selecionados para essas regiões. A resposta diferencial dos genótipos às mudanças ocorridas nos ambientes é chamada interação genótipos $\mathrm{X}$ ambientes (BOS \& CALIGARI, 1997). Uma das alternativas mais empregadas para minimizar a interação genótipos $\mathrm{x}$ ambientes é a identificação e indicação de cultivares pelo uso de metodologias apropriadas de adaptabilidade e estabilidade.
Contudo, apenas a correta utilização de metodologias de adaptabilidade e estabilidade não é suficiente, pois os resultados de indicação de cultivares podem estar influenciados pela precisão experimental, levando à diferença entre cultivares, que não são de natureza genética (CRUZ \& CARNEIRO, 2003). É importante, portanto, manter o erro experimental em níveis aceitáveis. Para tal, algumas medidas podem ser tomadas: a) o uso dos princípios básicos da experimentação no planejamento, na condução e na análise adequadas de experimentos (CRUZ \& CARNEIRO, 2003; STEEL et al., 1997); b) uso de maior número de repetições, juntamente com parcelas menores (CONAGIN et al., 1995; GOMES, 1994; STORCK et al., 2000); c) uso de unidades experimentais homogêneas (GOMES, 1990); d) realização, de maneira bastante uniforme, dos tratos culturais inerentes à cultura, no decorrer do experimento (LOPES \& STORCK, 1995).

\footnotetext{
${ }^{1}$ Engenheiro Agrônomo D. Sc. Centro de Ciências Agrárias da Universidade Federal do Espírito Santo/CCA - UFES - 29500-000 - Alegre, ES edilson@cca.ufes.br

${ }^{2}$ Engenheiro Agrônomo Mestrando em Genética e Melhoramento de Plantas - Departamento de Biologia - Universidade Federal de Lavras/UFLA Cx. P. 3037 - 37200-000 - Lavras, MG - willian.krause@pop.com.br

${ }^{3}$ Engenheiro Agrônomo D. Sc. Departamento de Biologia Geral - Universidade Federal de Viçosa/UFV - 36571-000 - Viçosa, MG - cdcruz@mail.ufv.br
} 
Apesar da busca de um menor erro experimental, salienta-se que os resultados experimentais podem ser influenciados pela perda de plantas competitivas por fatores que fogem ao controle do pesquisador, tais como elevada precipitação pluvial, ação de pragas e doenças (VENCOVSKY \& CRUZ, 1991). Segundo Steel et al. (1997), se não houver diferença estatística significativa entre o número de plantas na colheita (estande final) das cultivares avaliadas nos experimentos, deve-se fazer a correção da produtividade das parcelas usando como co-variável o estande final, por entender que as diferenças existentes entre o número de plantas não foram de natureza genética. Cruz \& Carneiro (2003) reforçam que o uso de co-variáveis, bem relacionadas à variável principal, com o estande, é uma boa opção para proceder à correção dos dados antes de submetê-los à análise de adaptabilidade e estabilidade.

A análise de co-variância com a produtividade de grãos de milho usando-se como co-variável o número de plantas na colheita (estande final) tem sido realizado por vários autores (CARGNELUTTI FILHO \& STORCK, 2004; MORAIS et al., 1986; SCHMILDT et al., 2001; VERONESI et al., 1995).

Existem outros métodos de correção da produtividade, como o método de Zuber (1942) e a correção usando regra de três. A fórmula de Zuber (1942) que, apesar de ser largamente utilizada por melhoristas, tem sido criticada: por não levar em consideração a disposição de falhas no campo; e pelo coeficiente de compensação pela falta de competição, que é valor fixo, igual a 0,3, independente da parcela (VERONESI et al., 1995). O uso de métodos que usam a regra de três para corrigir a produtividade em parcelas é impróprio por não existir proporcionalidade linear entre o número de plantas e a produtividade observada (SCHMILDT, 2000; VENCOVSKY \& CRUZ, 1991; VERONESI et al., 1995). Assim, uma das maneiras de se considerar o efeito de população em análise estatística é incluí-la como covariável.

Trabalhando com dados simulados, Vencovsky \& Cruz (1991) concluíram que o método de correção da produtividade de parcelas pelo método da covariância para o estande ideal foi o mais eficiente em qualquer situação estudada.

Segundo Schmildt (2000), considerando a existência de diferença estatística pelo teste $\mathrm{F}$ para as médias das cultivares com relação ao estande final por parcela, a indicação de não se fazer à correção da produtividade parece não ser totalmente apropriada, porém entende-se que se faz injusta a correção do rendimento das parcelas por um valor fixo do coeficiente de regressão $(b)$, como no método da co-variância. Para este caso, considera-se que o acréscimo por planta perdida, na realidade, não deverá ser necessariamente o mesmo para todas as cultivares, pois aqueles que não possuem habilidade para produção de estande ideal, poderiam não possuir o potencial semelhante de produtividade. Desde que o teste $\mathrm{F}$ tenha dado significativo para as médias do estande final por parcela, um método estatístico apropriado, permitirá que as cultivares sejam separadas em grupos e dessa forma corrigido cada grupo separadamente, ou seja, as cultivares serem separadas e corrigidas em estratos.

A aplicação de metodologias de correção da produtividade e a verificação da interferência dessas correções nas estimativas dos parâmetros da análise de adaptabilidade e estabilidade foi feita para a cultura do milho por Cargnelutti Filho \& Storck (2004), usando o método de Eberhart \& Russell (1966). Trabalhos dessa natureza empregando outros métodos de adaptabilidade e estabilidade de grande uso no momento não são conhecidos na literatura.

Desta forma, objetivou-se com este trabalho verificar o comportamento da precisão experimental pelo uso de métodos de correção de produtividade das parcelas experimentais e suas influências sobre a comparação e indicação de cultivares de milho pelo método de Annicchiarico (1992).

\section{MATERIAL E MÉTODOS}

Foram usados neste trabalho dados obtidos no Espírito Santo pelo Instituto Capixaba de Pesquisa, Assistência Técnica e Extensão Rural (INCAPER) e em Minas Gerais pela Universidade Federal de Viçosa (UFV), referente a ensaios regionais de competição de milho precoce (Zea mays L.), safra agrícola 1996/97. Foram analisados 33 cultivares de milho precoce utilizando o delineamento em blocos ao acaso, com 3 repetições, e repetidos em oito ambientes. Em todos os ensaios, as parcelas experimentais constaram de duas fileiras de 5,0 m, espaçadas de $0,90 \mathrm{~m}$, com área total por parcela de $9 \mathrm{~m}^{2}$.

O plantio foi realizado nos meses de outubro de 1996 e março de 1997. As adubações de plantio e de cobertura foram realizadas com base nas análises de solo e os tratos culturais e fitossanitários, de acordo com a necessidade da cultura.

No Espírito Santo foram instalados em Linhares, Sooretama e Cachoeiro de Itapemirim em outubro de 1996 e março de 1997. Em Minas Gerais, foram instalados dois ensaios em Coimbra, em outubro de 1996. 
Foi procedida uma análise de variância dos dados de produtividade e de estande final/parcela. Foi realizada a correção da produtividade em função do estande pela análise de covariância para o estande ideal (COVI), segundo Steel et al. (1997) e pela correção estratificada (CE) segundo Schmildt (2000), incluindo a análise sem correção (SC). Os métodos são descritos a seguir:

a) SC: sem correção. Nesse caso, os rendimentos foram submetidos à análise, desconsiderando-se a ocorrência de falhas nas parcelas, ou seja:

em que:

$$
\mathrm{Z}_{\mathrm{ij}}=\mathrm{Y}_{\mathrm{ij}}
$$

$\mathrm{Z}_{\mathrm{ij}}=$ produtividade corrigida para parcelas;

$\mathrm{Y}_{\mathrm{ij}}=$ rendimento observado nas parcelas;

b) COVI: correção na qual se utiliza a análise de covariância, com correção para o estande ideal. Esse processo tem sido bastante utilizado, principalmente na análise de grupos de experimentos, por permitir a estabilização dos valores ajustados ao redor do estande ideal, que deve ser comum a todos os ensaios. Assim, tem-se:

$$
\mathrm{Z}_{\mathrm{ij}}=\mathrm{Y}_{\mathrm{ij}}-\mathrm{b} \cdot\left(\mathrm{X}_{\mathrm{ij}}-\mathrm{H}\right)
$$

em que:

$Z_{\mathrm{ij}}$ e $\mathrm{Y}_{\mathrm{ij}}=$ descritos em a);

$\mathrm{X}_{\mathrm{ij}}=$ estande nas parcelas;

$\mathrm{H}=$ estande ideal que foi considerado como 50 plantas/ parcela;

$\mathrm{b}=$ coeficiente de regressão, que é dado por $\mathrm{b}=\frac{\operatorname{Cov}\left(\mathrm{X}_{\mathrm{ij}}, \mathrm{Y}_{\mathrm{ij}}\right)}{\widehat{\mathrm{V}}\left(\mathrm{X}_{\mathrm{ij}}\right)} \quad$, conforme Steel et al. (1997).

c) CE: correção estratificada. É baseada na correção pela análise de covariância para estande ideal e considera a obtenção de um coeficiente de regressão para cada grupo de cultivares. Os grupos de cultivares foram estabelecidos nos ambientes cujo teste $\mathrm{F}$ tenha sido significativo, para a característica estande final/parcela.

em que:

$$
\mathrm{Z}_{\mathrm{ijk}}=\mathrm{Y}_{\mathrm{ijk}}-\mathrm{b}_{\mathrm{k}} \cdot\left(\mathrm{X}_{\mathrm{ijk}}-\mathrm{H}\right)
$$

$\mathrm{Z}_{\mathrm{ijk}}=$ rendimento corrigido para a i-ésima cultivar na j-ésima repetição, no k-ésimo grupo, estabelecido com base na inexistência de diferença significativa entre as médias, conforme teste de Scott \& Knott (1974), realizado em nível de 5\% de significância;

$\mathrm{Y}_{\mathrm{ijk}}=$ produtividade antes da correção, para a i-ésima cultivar na j-ésima repetição e no k-ésimo grupo; e $\mathrm{b}_{\mathrm{k}}=$ valor de coeficiente de regressão para o k-ésimo grupo ( $\mathrm{k}=1,2, \ldots \mathrm{n}$, sendo $\mathrm{n}$ o número de grupos).

Nas comparações da eficiência relativa de cada técnica de ajuste, para cada ambiente, utilizaram-se como parâmetros o coeficiente de variação $(\mathrm{CV})$ e os valores da estatística F, calculada para avaliar a significância dos tratamentos, conforme Veronesi et al. (1995), que admitem como melhor método de ajuste aquele que: 1) minimize as variações de produção, devidas a estandes variados entre as parcelas de um mesmo tratamento, proporcionando baixos valores do CV; 2) corrija para fatores ambientais e não altere as variações genéticas refletidas pelas diferenças entre médias de tratamentos e, assim, não provoque reduções nos valores de $F$.

Considerando a análise conjunta de ambientes, utilizou-se o método de ajuste proposto por Schmildt (2000) que permite a obtenção dos menores valores de $\mathrm{CV}$ e maiores de $\mathrm{F}$, constantes nos vários ambientes. Nesse método, para cada estatística isoladamente ou para ambas conjuntamente, faz-se uso dos princípios da metodologia para adaptabilidade e estabilidade de Lin \& Binns (1988):

1 - para cada estatística (CV ou F):

$$
\mathrm{P}_{\mathrm{i}}=\frac{\sum_{\mathrm{j}=1}^{\mathrm{n}}\left(\mathrm{W}_{\mathrm{ij}}-\mathrm{M}_{\mathrm{j}}\right)^{2}}{2 \mathrm{e}}
$$

em que:

$\mathrm{P}_{\mathrm{i}}=$ estimativa da eficácia do método i. Quanto menor o valor de $\mathrm{P}_{\mathrm{i}}$, mais eficaz é o método;

$\mathrm{W}_{\mathrm{ij}}=$ valor de 100/CV ou $\mathrm{F}$ do i-ésimo método no j-ésimo ambiente, sendo que $\mathrm{P}_{\mathrm{i}}$ torna-se $\mathrm{P}_{\mathrm{i}(100 / \mathrm{CV})}$ e $\mathrm{P}_{\mathrm{i}(\mathrm{F})}$, respectivamente. O valor $100 / \mathrm{CV}$ foi utilizado para que se tome como melhor método aquele de menor $\mathrm{P}_{\mathrm{i}}$, nas duas estatísticas de comparação (CV e F);

$\mathrm{M}_{\mathrm{j}}$ é a resposta máxima de $100 / \mathrm{CV}$ para $\mathrm{P}_{\mathrm{i}(100 / \mathrm{CV})}$ ou F para $\mathrm{P}_{\mathrm{i}(\mathrm{F})}$ entre todos os métodos no ambiente $\mathrm{j} ; \mathrm{e}$

e = número de ambientes.

2 - Multivariado, conforme o princípio da medida de adaptabilidade e estabilidade de comportamento de cultivares adotado por Carneiro (1998):

$$
\mathrm{P}_{\mathrm{mi}}=\sum_{\mathrm{k}=1}^{\mathrm{v}}\left[\mathrm{P}_{\mathrm{ik}} \frac{1}{\hat{\sigma}_{\mathrm{pk}}}\right]
$$

Ciênc. agrotec., Lavras, v. 30, n. 1, p. 72-80, jan./fev., 2006 
em que:

$\mathrm{P}_{\mathrm{mi}}=$ estimador do $\mathrm{P}_{\mathrm{i}}$ multivariado para o método de ajuste $\mathrm{i}$; $\mathrm{P}_{\mathrm{ik}}=$ estimador do $\mathrm{P}_{\mathrm{i}}$ para cada método i relativo à k-ésima estatística (coeficiente de variação ou F); e $\hat{\sigma}_{\mathrm{pk}}=$ desvio padrão dos $\mathrm{P}_{\mathrm{i}}$ 's para a k-ésima estatística.

Os dados de produtividade, sem ajuste (SC) e os ajustados pelos métodos COVI e CE foram analisados para avaliação da adaptabilidade e estabilidade pelo método de Annicchiarico (1992) para observar se houve influência sobre a indicação das cultivares.

Os procedimentos para os cálculos pelo método proposto por Annicchiarico (1992) dão-se, inicialmente, com a transformação das médias de cada cultivar em cada ambiente, em porcentagem da média do ambiente. Posteriormente, estima-se a média $\left(\bar{Y}_{i}\right)$ e desvio padrão $\left(\hat{\sigma}_{i}\right)$ das porcentagens de cada cultivar. De posse dessas estimativas, obtém-se o índice de confiança $\left(I_{i}\right)$ por meio do seguinte estimador:

$$
I_{i}=\bar{Y}_{i .}-Z_{(1-\alpha)}\left(\hat{\sigma}_{i .}\right)
$$

sendo ainda

$Z_{(1-\alpha)}=$ valor na distribuição normal estandardizada no qual a função de distribuição acumulada atinge o valor $(1-\alpha)$, com nível de significância $\alpha$ pré-fixado pelo autor em 0,25 .

Quanto maior for o índice de confiança, maior será a confiança na recomendação do cultivar.

Foi determinada a correlação classificatória de Spearman entre os índices de confiança de Annicchiarico (1992) obtidos pelos métodos de correção da produtividade.

As análises foram procedidas com o auxílio do programa GENES (CRUZ, 2003) e do aplicativo Office Excel.

\section{RESULTADOS E DISCUSSÃO}

As análises estatísticas referentes a estande final nos oito ambientes avaliados são apresentadas na Tabela 1. A média do estande final por parcela apresentou redução em relação ao estande ideal (50 plantas/parcela) em todos os ambientes. A redução variou de $0,44 \%$, no ambiente 7 , até $11,44 \%$, no ambiente 5 . O coeficiente de variação variou de $3,92 \%$ no ambiente 4 até $12,82 \%$ no ambiente 5 , sendo considerado médio nesse ambiente e baixo nos demais, segundo Gomes (1990) para dados de campo, demonstrando bom controle do erro experimental. Há heterogeneidade de variâncias residuais, em que se pode observar que o quadrado médio do resíduo está variando de 3,8 até 32,2, realçando os efeitos de ambientes e da interação genótipos $\mathrm{x}$ ambientes. Detectou-se diferença significativa entre as médias das cultivares pelo teste $\mathrm{F}$ para os ambientes 1, 3, 4 e 6, demonstrando comportamento diferencial das cultivares nos ambientes. Verificou-se, também, que a significância, ou não, pelo teste $\mathrm{F}$ independe da amplitude da variação em relação ao estande ideal. Observa-se, assim, que tanto o ambiente que apresentou maior redução quanto o que apresentou menor redução foram não-significativos pelo teste F. O mesmo comportamento foi observado em relação ao coeficiente de variação experimental. Assim, verifica-se que tanto o ambiente 5, de maior coeficiente de variação experimental (12,82\%), quanto os ambientes 8 e 7 , de segundo e terceiro menor coeficiente de variação experimental (4,25 e 4,37), apresentaram-se como nãosignificativos. Assim, apesar da boa precisão experimental, a desuniformidade na população de plantas, pode ser uma das causas da superioridade de produtividade de uma cultivar em relação a outra, superioridade esta que não é de natureza genética, justificando a adoção de medidas de correção (CRUZ \& CARNEIRO, 2003).

TABELA 1 - Média, coeficiente de variação (CV), quadrados médios de cultivares e de resíduo e teste F para a característica estande final/parcela na produção de milho, em oito ambientes.

\begin{tabular}{|c|c|c|c|c|c|c|}
\hline \multirow{3}{*}{ Ambiente } & \multicolumn{6}{|c|}{ Estande Final/Parcela } \\
\hline & \multirow{2}{*}{ Média } & \multirow{2}{*}{$\mathrm{CV}(\%)$} & \multicolumn{2}{|c|}{$\mathbf{Q M}$} & \multirow{2}{*}{\multicolumn{2}{|c|}{$\mathbf{F}$}} \\
\hline & & & Cultivares & Resíduo & & \\
\hline 1 & 47,88 & 5,45 & 16,18 & 6,81 & 2,37 & ** \\
\hline 2 & 48,11 & 6,47 & 14,56 & 9,70 & 1,50 & $\mathrm{~ns}$ \\
\hline 3 & 46,35 & 6,10 & 30,27 & 7,99 & 3,79 & ** \\
\hline 4 & 49,46 & 3,92 & 9,17 & 3,77 & 2,43 & ** \\
\hline 5 & 44,28 & 12,82 & 43,84 & 32,23 & 1,36 & ns \\
\hline 6 & 48,61 & 6,80 & 19,28 & 10,92 & 1,77 & * \\
\hline 7 & 49,78 & 4,37 & 5,31 & 4,74 & 1,12 & ns \\
\hline 8 & 48,55 & 4,25 & 6,18 & 4,27 & 1,45 & ns \\
\hline
\end{tabular}

ns Não-significativo. * ** Significativo a 5 e $1 \%$ de probabilidade, respectivamente, pelo teste $\mathrm{F}$. 
Os valores de coeficiente de variação, para produtividade, após correção ou não dos dados, são apresentados na Tabela 2. Esses valores são considerados baixos para os ambientes 1, 3, 4 e 6 e médios para os demais ambientes segundo classificação de Gomes (1990) e Scapim et al. (1995). O método sem correção (SC) proporcionou o maior valor do coeficiente de variação em todos os experimentos, o que pode ser comprovado pelo maior valor de $\mathrm{P}_{\mathrm{i}(10 / \mathrm{CV})}$, ou seja 0,9751 . Nota-se também que em todos os ambientes houve redução no coeficiente de variação após correção da produtividade, atingindo até $36,92 \%$ no ambiente 5 , tanto para o método da covariância para estande ideal (COVI) quanto no método da correção estratificada (CE). Como conseqüência, ocorre aumento na precisão experimental $\mathrm{e}$, melhoria na eficiência dos experimentos.

Nos ambientes 2, 5, 7 e 8, cujo teste F não apontou diferenças significativas entre as médias de estandes das cultivares (Tabela 1), os valores dos coeficientes de variação dos métodos COVI e CE, passam a apresentar os mesmos resultados. Nos ambientes 1, 3, 4 e 6 , cujo teste $F$ apontou diferenças significativas entre as médias de estandes das cultivares, foi feita estratificação dos ambientes, pelo teste de Scott \& Knott (1974). Nesses quatro ambientes, os valores dos coeficientes de variação foram reduzidos pela correção COVI e CE, cada um sendo melhor em dois ambientes (Tabela 2). Considerando os valores de $\mathrm{P}_{\mathrm{i}(100 /}$ cv), que medem a magnitude do coeficiente de variação considerando todos os ambientes, estes são de 0,0005 para o método COVI e de 0,0030 para o método CE. Nota-se, portanto, que a correção pelos métodos COVI e CE aumentou consideravelmente a precisão dos experimentos, ajustando bem os dados para análise conjunta, o que também foi observado por Cargnelutti Filho \& Storck (2004) trabalhando com a mesma cultura.

Os valores do teste $\mathrm{F}$ para produtividade após correção ou não dos dados são apresentados na Tabela 3. Observa-se que a análise dos dados sem correção prévia proporcionou os maiores valores do teste $\mathrm{F}$ em cinco ambientes. $\mathrm{O}$ método $\mathrm{CE}$ proporcionou maior valor de $\mathrm{F}$ nos outros três ambientes. Observa-se, ainda, que nos ambientes em que não se apresentou com o maior valor de $\mathrm{F}$, o método CE teve valores altos, bem próximos aos maiores observados, o que pode resultar no baixo valor de $\mathrm{P}_{\mathrm{i}(\mathrm{F})}=0,0406$.

Apesar de o método CE ter apresentado a segunda colocação em termos de eficácia para as estatísticas $\mathrm{P}_{\mathrm{i}(100 / \mathrm{CV})} \mathrm{e}$
$\mathrm{P}_{\mathrm{i}(\mathrm{FF}}$, ele foi o método mais apropriado, considerando os dois parâmetros simultaneamente, pela estatística $\mathrm{P}_{\mathrm{mi}}$ (Tabela 3). Tal fato pode, em parte, ser explicado pelo comportamento dos métodos de correção em relação aos dois parâmetros isoladamente. Assim, o método COVI, que foi o mais eficiente com relação ao parâmetro $P_{\text {i }(100 / \mathrm{CV})}$, ocupou a última posição em relação ao parâmetro $\mathrm{P}_{\mathrm{i}(\mathrm{F})}$ e o método $\mathrm{SC}$, que foi o mais eficaz com relação a esse parâmetro, foi o menos eficaz em relação ao parâmetro $\mathrm{P}_{\mathrm{i}(100 / \mathrm{CV})^{*}}$.

$\mathrm{Na}$ Tabela 4, apresenta-se os resultados referentes à média de produtividade e índice de confiança de Annicchiarico (1992) após correção ou não dos dados. Verifica-se que a correção dos dados, tanto pelo método COVI como pelo método $\mathrm{CE}$, provocou alterações nas médias e no índice de confiança. Analisando os índices de confiança pelo método de Annicchiarico (1992), verificase que os valores variaram de $73 \%$ para a cultivar DENSUS até $119,17 \%$ para a cultivar XHT20 pelo método SC. Esses valores são o menor e o maior valor comparando com os métodos COVI e CE. Com isso verifica-se que houve alteração nos valores do índice de confiança com a correção da produtividade, mesmo com a correlação classificatória de Spearmam sendo alta (aproximadamente 96\%) entre os métodos de correção e o método sem correção. Os métodos de correção COVI e CE apresentaram correlação de Spearman de 0,998\%. Cargnelutti Filho \& Storck (2004) também verificaram boa concordância na indicação de cultivares pelos métodos COVI e CE, usando o método de adaptabilidade e estabilidade de Eberhart \& Russell (1966). Segundo Cargnelutti Filho \& Storck (2004), a correção interfere na média, na medida de adaptabilidade e na de estabilidade, sendo mais acentuada nesta última. Para o método de Annicchiarico (1992), quanto maior o índice de confiança, maior será a estabilidade, traduzida na confiança da indicação da cultivar (SCHMILDT, 2000). Assim, é possível verificar que a correção tanto pelo método COVI quanto pelo método $\mathrm{CE}$ provocaram alterações na estabilidade das cultivares e, por conseguinte na escolha dos que seriam indicados. Considerando a indicação das cinco cultivares mais estáveis, pelo método SC seriam XHT 20, P 3041, DINA 657, DINA 766 e XL 360 (Tabela 4). Para os métodos COVI e CE seriam indicados apenas os três primeiros do método SC. Considerando a consistência dos métodos COVI e CE, as doze primeiras cultivares, com índice de confiança superior a $100 \%$ pelo método COVI apresentamse na mesma sequiência também pelo método CE. 
TABELA 2 - Valores do coeficiente de variação percentual na produtividade de grãos de milho (kg/ha) após correção ou não, em função de falhas na parcela, por diferentes métodos.

\begin{tabular}{cccccc}
\hline \multirow{2}{*}{ Ambiente } & \multicolumn{5}{c}{ Método de Correção $^{\mathbf{1 /}}$} \\
\cline { 2 - 6 } & $\mathbf{S C}$ & COVI & \% redução CV & CE & \% redução CV \\
\hline 1 & 7,78 & 7,78 & 0,00 & 7,75 & 0,39 \\
2 & 11,94 & 10,19 & 14,66 & 10,19 & 14,66 \\
3 & 8,94 & 7,64 & 14,54 & 7,60 & 14,99 \\
4 & 9,34 & 9,07 & 2,89 & 9,18 & 1,71 \\
5 & 19,93 & 12,72 & 36,92 & 12,72 & 36,92 \\
6 & 9,31 & 8,49 & 8,81 & 8,61 & 7,52 \\
7 & 13,54 & 13,46 & 0,59 & 13,46 & 0,59 \\
8 & 16,01 & 14,50 & 9,43 & 14,50 & 9,43 \\
$\mathrm{P}_{\mathrm{i}(100 / \mathrm{CV})}$ & 0,9751 & 0,0005 & - & 0,0030 & - \\
\hline
\end{tabular}

${ }^{1 /} \mathrm{SC}=$ sem correção; $\mathrm{COVI}=$ correção pela co-variância com estande ideal; $\mathrm{CE}=$ correção estratificada.

TABELA 3 - Valores da estatística F na produção de grãos de milho $(\mathrm{kg} / \mathrm{ha})$ após correção ou não, em função de falhas na parcela, por diferentes métodos.

\begin{tabular}{cccc}
\hline \multirow{2}{*}{ Ambiente } & \multicolumn{3}{c}{ Método de Correção $^{\mathbf{1 /}}$} \\
\cline { 2 - 4 } & $\mathbf{S C}$ & COVI & CE \\
\hline 1 & 13,83 & 13,81 & 14,53 \\
2 & 4,90 & 4,64 & 4,64 \\
3 & 8,53 & 7,07 & 8,02 \\
4 & 2,81 & 2,91 & 2,93 \\
5 & 1,32 & 1,43 & 1,43 \\
6 & 5,33 & 5,10 & 4,81 \\
7 & 2,25 & 2,20 & 2,20 \\
8 & 2,06 & 1,89 & 1,89 \\
$\mathrm{P}_{\mathrm{i}(\mathrm{F})}$ & 0,0358 & 0,1764 & 0,0406 \\
$\mathrm{P}_{\mathrm{mi}}$ & 25,316 & 22,831 & 22,703 \\
\hline
\end{tabular}

${ }^{1 /} \mathrm{SC}=$ sem correção; $\mathrm{COVI}=$ correção pela co-variância com estande ideal e CE = correção estratificada. 
TABELA 4 - Estimativas das médias de produtividade de milho ( $\mathrm{kg} / \mathrm{ha})$ após correção ou não da produtividade e índice de confiança $\left(\mathrm{I}_{\mathrm{i}}\right)$ conforme o método de Annicchiarico (1992).

\begin{tabular}{|c|c|c|c|c|c|c|}
\hline \multirow{3}{*}{ Cultivar } & \multicolumn{6}{|c|}{ Métodos de correção $^{1 /}$} \\
\hline & \multicolumn{2}{|c|}{ SC } & \multicolumn{2}{|c|}{ COVI } & \multicolumn{2}{|r|}{$\mathbf{C E}$} \\
\hline & Média & $\mathbf{I}_{\mathbf{i}}{ }^{2 /}$ & Médi & $\mathbf{I}_{\mathbf{i}}^{2 /}$ & & $\mathbf{I}_{\mathbf{i}}{ }^{\prime \prime}$ \\
\hline XHT 20 & 7.789 & $119,17(01)$ & 7.878 & $116,24(01)$ & 7.896 & $116,53(01)$ \\
\hline P 3041 & 7.517 & $114,21(02)$ & 7.586 & $111,79(02)$ & 7.586 & $111,80(02)$ \\
\hline DINA 657 & 7.074 & $106,95(03)$ & 7.292 & $106,63(03)$ & 7.285 & $106,27(03)$ \\
\hline DINA 766 & 6.899 & $105,55(04)$ & 7.113 & $105,04(05)$ & 7.123 & $105,18(05)$ \\
\hline XL 360 & 6.850 & $104,44(05)$ & 6.950 & $101,76(08)$ & 6.965 & $101,92(08)$ \\
\hline MASTER & 6.791 & $103,20(08)$ & 7.131 & $106,02(04)$ & 7.126 & $105,88(04)$ \\
\hline XHT 12 & 6.786 & $103,68(07)$ & 6.913 & $102,05(07)$ & 6.947 & $102,46(07)$ \\
\hline AGX 5482 & 6.785 & $103,79(06)$ & 7.044 & $103,80(06)$ & 7.056 & $103,76(06)$ \\
\hline ZEN 83E92 & 6.747 & $101,25(10)$ & 6.867 & $99,55(14)$ & 6.883 & $99,72(14)$ \\
\hline P 3071 & 6.721 & $102,01(09)$ & 6.867 & $100,77(11)$ & 6.865 & $100,54(11)$ \\
\hline BR 3123 & 6.701 & $100,21(11)$ & 6.978 & $101,63(09)$ & 6.961 & $101,17(09)$ \\
\hline $\mathrm{CO} 32$ & 6.609 & $100,11(13)$ & 6.814 & $100,78(10)$ & 6.809 & $100,69(10)$ \\
\hline CO 34 & 6.586 & $100,13(12)$ & 6.741 & $98,72(17)$ & 6.691 & $98,59(17)$ \\
\hline M 9560 & 6.582 & $99,61(14)$ & 6.697 & $98,18(18)$ & 6.692 & 98,39 (18) \\
\hline ZEN 83E00 & 6.566 & $98,74(16)$ & 6.827 & $99,33(16)$ & 6.845 & $99,54(16)$ \\
\hline XL 221 & 6.561 & $98,33(17)$ & 6.799 & 99,64 (13) & 6.832 & $100,14(13)$ \\
\hline XL 345 & 6.518 & $99,13(15)$ & 6.776 & $99,36(15)$ & 6.764 & $99,62(15)$ \\
\hline ZEN 84E74 & 6.512 & $97,41 \quad(19)$ & 6.873 & $100,25(12)$ & 6.880 & $100,29(12)$ \\
\hline C 909 & 6.505 & $98,02(18)$ & 6.578 & $96,27(20)$ & 6.587 & $96,48(19)$ \\
\hline AG 5011 & 6.374 & $94,99(20)$ & 6.534 & $94,07 \quad(23)$ & 6.467 & $93,31(25)$ \\
\hline EXCELER & 6.320 & $94,48(22)$ & 6.512 & $94,91(21)$ & 6.523 & $95,06(21)$ \\
\hline AG 5014 & 6.231 & $93,79(24)$ & 6.394 & $92,88(25)$ & 6.426 & $93,39(24)$ \\
\hline AGROMEN & & $94,81 \quad(21)$ & 6.373 & 94,04 (24) & 6.386 & $94,38(23)$ \\
\hline 2012 & 6.216 & & & & & \\
\hline C 806 & 6.214 & $93,92(23)$ & 6.678 & 98,11 (19) & 6.562 & $96,05(20)$ \\
\hline AGX 9674 & 6.069 & $92,41 \quad(25)$ & 6.376 & $94,20(22)$ & 6.395 & $94,40(22)$ \\
\hline AGROMEN & & $90,28(27)$ & 6.272 & $91,11(26)$ & 6.252 & $90,78(26)$ \\
\hline 3000 & 6.033 & & & & & \\
\hline AL 25 & 6.001 & 90,45 (26) & 6.194 & $90,52(27)$ & 6.202 & 90,49 (28) \\
\hline DINA 769 & 5.869 & $89,22(28)$ & 6.132 & $90,47 \quad(28)$ & 6.142 & $90,60(27)$ \\
\hline AGX 5273 & 5.819 & 87,63 (29) & 6.075 & $88,62(30)$ & 6.095 & $88,90(30)$ \\
\hline AG 9014 & 5.646 & $83,34(31)$ & 5.795 & $88,07 \quad(31)$ & 5.797 & $82,82(32)$ \\
\hline ZEN 84E90 & 5.603 & $82,67 \quad(32)$ & 6.134 & $87,84(32)$ & 6.095 & $87,10(31)$ \\
\hline AG 3010 & 5.585 & $84,78(30)$ & 6.116 & 89,49 (29) & 6.111 & $89,48 \quad(29)$ \\
\hline DENSUS & 5.003 & $73,00 \quad(33)$ & 5.411 & 80,29 (33) & 5.399 & 80,07 (33) \\
\hline Média & 6.426 & - & 6.655 & - & 6.656 & - \\
\hline
\end{tabular}

${ }^{1 /} \mathrm{SC}=$ sem correção; COVI = correção pela co-variância com estande ideal e CE = correção estratificada.

${ }^{2 /}$ Números entre parênteses indicam a posição relativa de indicação das cultivares a partir do índice de confiança de Annicchiarico (1992), tendo os valores da correlação de Spearman entre os métodos: $\mathrm{r}_{(\mathrm{SCxCOVI})}=0,9636 ; \mathrm{r}_{(\mathrm{SCXCE})}=0,9626$; $\mathrm{r}_{\text {(COVIXCE) }}=0,9980$. 


\section{CONCLUSÕES} cultivares;

a) A correção dos dados influencia na indicação de

b) Houve redução no coeficiente de variação, com o ajuste da produtividade, melhorando a eficiência dos experimentos;

c) Os métodos da covariância e correção estratificada foram consistentes na correção da produtividade para indicação de cultivares de milho.

\section{REFERÊNCIAS BIBLIOGRÁFICAS}

ANNICCHIARICO, P. Cultivar adaptation and recomendation from alfafa trials in Northern Italy. Journal of Genetics and Plant Breeding, Berlin, v. 46, p. 269-278, 1992.

BOS, I.; CALIGARI, P. Selection methods in plant breeding. London: Chapman \& Hall, 1997. 347 p.

CARGNELUTTI FILHO, A.; STORCK, L. Métodos de correção da produtividade de grãos de milho relacionado à adaptabilidade e estabilidade de cultivares. Pesquisa Agropecuária Brasileira, Brasília, v. 39, n. 9, p. 857-864, 2004.

CARNEIRO, P. C. S. Novas metodologias de análise da adaptabilidade e estabilidade de comportamento. 1998. 168 f. Dissertação (Doutorado em Genética e Melhoramento) Universidade Federal de Viçosa, Viçosa, 1998.

CONAGIN, A.; IGUE, T.; NAGAI, V. Tabelas para determinação do número de repetições no planejamento de experimentos. Campinas: Instituto Agronômico de Campinas, 1995. 17 p. (Boletim científico, 34).

CRUZ, C. D. Programa GENES, aplicativo computacional em genética e estatística. Viçosa: UFV, 2003. CD-ROM.

CRUZ, C. D.; CARNEIRO, P. C. S. Modelos biométricos aplicados ao melhoramento de plantas. Viçosa: UFV, 2003. $585 \mathrm{p}$.

EBERHART, S. A.; RUSSELL, W. A. Stability parameters for comparing varieties. Crop Science, Madison, v. 6, n. 1, p. $36-40,1966$.

GOMES, F. P. Curso de estatística experimental. 13. ed. Piracicaba: Nobel, 1990. 401 p.
GOMES, F. P. A importância do número de repetições nos experimentos. Revista de Agricultura, Piracicaba, v. 69, n. 3, p. 243-245, 1994.

LIN, C. S.; BINNS, M. R. A superiority measure of cultivar performance for cultivar $\mathrm{x}$ location data. Canadian Journal of Plant Science, Ottawa, v. 68, n. 3, p. 193-198, 1988.

LOPES, S. J.; STORCK, L. A precisão experimental para diferentes manejos na cultura do milho. Ciência Rural, Santa Maria, v. 25, n. 1, p. 49-53, 1995.

MORAIS, A. R.; OLIVEIRA, A. C.; CRUZ, J. C. Correção de produções de grãos de milho em parcelas experimentais. In:__. Relatório técnico anual do CNPMS - 19801984. Sete Lagoas: CNPMS, 1986. p. 130-132.

SCAPIM, A.; CARVALHO, C. G. P.; CRUZ, C. D. Uma proposta de classificação dos coeficientes de variação para a cultura do milho. Pesquisa Agropecuária Brasileira, Brasília, v. 30, n. 5, p. 683-686, 1995.

SCHMILDT, E. R. Correção de rendimento de parcelas, estratificação ambiental e adaptabilidade e estabilidade de cultivares de milho. 2000. $111 \mathrm{f}$. Tese (Doutorado em Genética e Melhoramento de Plantas) - Universidade Federal de Viçosa, Viçosa, 2000.

SCHMILDT, E. R.; CRUZ, C. D.; ZANUNCIO, J. C.; PEREIRA, P. R. G.; FERRÃO, R. G. Avaliação de métodos de correção do estande para estimar a produtividade em milho. Pesquisa Agropecuária Brasileira, Brasília, v. 36, n. 8 , p. 1011-1018, 2001.

SCOTT, A. J.; KNOTT, M. A. A cluster analysis method for grouping means in the analysis of variance. Biometrics, Washington, v. 30, p. 507-512, 1974.

STEEL, R. G. D.; TORRIE, J. H.; DICKEY, D. Principles and procedures of statistics: a biometrical approach. 3. ed. Boston: McGraw-Hill, 1997. 666 p.

STORCK, L.; GARCIA, D. C.; LOPES, S. J. Experimentação vegetal. Santa Maria: UFSM, 2000. 198 p.

VENCOVSKY, R.; CRUZ, C. D. Comparação de métodos de correção de rendimento de parcelas experimentais com estandes variados: I. dados simulados. Pesquisa Agropecuária Brasileira, Brasília, v. 26, p. 647-657, 1991. 
VERONESI, J. A.; CRUZ, C. D.; CORRÊA, L. A.; SCAPIM, C. A. Comparação de métodos de ajuste do rendimento de parcelas com estandes variados. Pesquisa Agropecuária Brasileira, Brasília, v. 30, p. 169-174, 1995.
ZUBER, M. S. Relative efficiency of incomplete block designs using corn uniform trial data. Journal of the American Society of Agronomy, [S.1.], v. 34, p. 30-47, 1942.

Ciênc. agrotec., Lavras, v. 30, n. 1, p. 72-80, jan./fev., 2006 